\title{
Propagation of Cross-Section Uncertainties in Criticality Calculations in the Framework of UAM-Phase I Using MCNPX-2.7e and SCALE-6.1
}

\author{
C. J. Díez, ${ }^{1}$ J. J. Herrero, ${ }^{1}$ O. Cabellos, ${ }^{1,2}$ and J. S. Martínez ${ }^{1}$ \\ ${ }^{1}$ Department of Nuclear Engineering, Universidad Politéenica de Madrid, C/José Gutiérrez Abascal 2, 28006 Madrid, Spain \\ ${ }^{2}$ Institute of Nuclear Fusion, Universidad Politécnica de Madrid, C/José Gutiérrez Abascal 2, 28006 Madrid, Spain
}

Correspondence should be addressed to C. J. Díez; cj.diez@upm.es

Received 22 August 2012; Accepted 7 December 2012

Academic Editor: Kostadin Ivanov

Copyright (C) 2013 C. J. Díez et al. This is an open access article distributed under the Creative Commons Attribution License, which permits unrestricted use, distribution, and reproduction in any medium, provided the original work is properly cited.

In the framework of the OECD/NEA project on Benchmark for Uncertainty Analysis in Modeling (UAM) for Design, Operation, and Safety Analysis of LWRs, several approaches and codes are being used to deal with the exercises proposed in Phase I, "Specifications and Support Data for Neutronics Cases." At UPM, our research group treats these exercises with sensitivity calculations and the "sandwich formula" to propagate cross-section uncertainties. Two different codes are employed to calculate the sensitivity coefficients of $k_{\text {eff }}$ to cross sections in criticality calculations: MCNPX-2.7e and SCALE-6.1. The former uses the Differential Operator Technique and the latter uses the Adjoint-Weighted Technique. In this paper, the main results for exercise I-2 "Lattice Physics" are presented for the criticality calculations of PWR. These criticality calculations are done for a $15 \times 15$ TMI fuel assembly at four different states: HZP-Unrodded, HZP-Rodded, HFP-Unrodded, and HFP-Rodded. The results of the two different codes above are presented and compared. The comparison proves a good agreement between SCALE-6.1 and MCNPX-2.7e in $k_{\text {eff }}$ uncertainty that comes from the sensitivity coefficients calculated by both codes. Differences are found when the sensitivity profiles are analysed, but they do not lead to differences in the $k_{\text {eff }}$ uncertainty.

\section{Introduction}

As stated in the Introduction of [1], "This benchmark project (...) responds to the needs of estimating confidence bounds for the results from simulations and analysis in real applications." Phase I deals with the neutronics cases.

(i) Exercise I-1. Cell Physics focused on the derivation of the multigroup microscopic cross section libraries and their uncertainties.

(ii) Exercise I-2. Lattice Physics focused on the derivation of the few-group macroscopic cross section libraries and their uncertainties.

(iii) Exercise I-3. Core Physics focused on the core steady state stand-alone neutronics calculations and their uncertainties.

For these calculations, the main source of uncertainty taken into account is the cross section uncertainties which are propagated throughout the different simulation levels.
There are mainly two different approaches to propagate uncertainties: The first one is based on a Monte Carlo approach where a large amount of calculations are performed sampling the problem parameters as random variables, and then carrying out a statistical analysis; the second one relies on sensitivity coefficients and the "sandwich formula." The latter approach is the one employed in this work.

The way of obtaining the sensitivity coefficients of the response functions depends on which code is used. In this case, two different techniques are used: the Adjoint-Weighted Technique by SCALE-6.1 [2] and the Differential Operator Technique by MCNPX-2.7e [3]. Previous works [4, 5] have compared both techniques showing their pros and cons.

This work is aimed to present how the uncertainty quantification is carried out using the sensitivity approach and how the sensitivity coefficients are calculated with SCALE6.1 and MCNPX-2.7e. Afterwards, in the framework of Exercise I-2, both codes are used to perform the uncertainty quantification on the $k_{\text {eff }}$ of a $15 \times 15$ TMI fuel assembly. 
Finally, their results are compared and conclusions are drawn.

\section{Uncertainty Quantification Based on Sensitivity Coefficients}

The uncertainty quantification based on sensitivity coefficients relies on the "sandwich formula" obtained with the propagation of moments, as presented in [6, Section III.F] and summarized here.

Being $R$ the calculated response function of a system which depends on $\left(\alpha_{1}, \ldots, \alpha_{n}\right)$ parameters, $R$ can be approximated by a linear function of $\left(\alpha_{1}, \ldots, \alpha_{n}\right)$ using the Taylor series approximation around a nominal value $\left(\alpha_{1}^{0}, \ldots, \alpha_{n}^{0}\right)$, as presented in (1), where $\delta \alpha_{i}=\alpha_{i}-\alpha_{i}^{0}$ :

$$
R\left(\alpha_{1}, \ldots, \alpha_{n}\right)=R\left(\alpha_{1}^{0}, \ldots, \alpha_{n}^{0}\right)+\sum_{i=1}^{n}\left(\frac{\partial R}{\partial \alpha_{i}}\right)_{\left(\alpha_{1}^{0}, \ldots, \alpha_{n}^{0}\right)} \delta \alpha_{i}
$$

Then, taking the parameters of the system as random variables, $R$ becomes a random variable for which its mean coincides with the response function at the nominal value, (2), and its variance is calculated with (3) known as the "sandwich formula" or the "sandwich rule":

$$
\begin{gathered}
E(R)=R\left(\alpha_{1}^{0}, \ldots, \alpha_{n}^{0}\right), \\
\operatorname{var}(R)=S V_{\alpha} S^{T},
\end{gathered}
$$

where

(i) $S=\left(\partial R / \partial \alpha_{1}, \ldots, \partial R / \partial \alpha_{n}\right)$ is the vector of the sensitivity coefficients.

(ii) $V_{\alpha}$ is the covariance matrix of the system parameters defined as

$$
\left(V_{\alpha}\right)_{i j}= \begin{cases}\operatorname{cov}\left(\alpha_{i}, \alpha_{j}\right), & i \neq j \\ \operatorname{var}\left(\alpha_{i}\right), & i=j\end{cases}
$$

Because usually the sensitivity coefficients are calculated as relative values,

$$
S_{i}^{\prime}=\frac{\partial R / R}{\partial \alpha_{i} / \alpha_{i}} .
$$

Equation (3) can be rewritten as (6), where the value obtained is the relative standard deviation (rel.std.dev. $(R))$ and $V_{\alpha}^{\prime}$ is the relative covariance matrix:

$$
\begin{aligned}
(\text { rel.std.dev. }(R))^{2} & =\frac{\operatorname{var}(R)}{R^{2}} \\
& =\sum_{i=1, j=1}^{n}\left(S_{i}^{\prime} \frac{\operatorname{cov}\left(\alpha_{i}, \alpha_{j}\right)}{\alpha_{i} \alpha_{j}} S_{j}^{\prime}\right)=S^{\prime} V_{\alpha}^{\prime} S^{\prime T} .
\end{aligned}
$$

Both values, the standard deviation $\sqrt{\operatorname{var}(R)}$ provided by (3) and the rel.std.dev. $(R)$ calculated in (6), are used as a measure of the uncertainty on the response function. Therefore, the sensitivity coefficients of the response function to the system parameters, $S_{i}$ or $S_{i}^{\prime}$, should be calculated and the covariance matrix $\left(V_{\alpha}\right)$ or the relative covariance matrix $\left(V_{\alpha}^{\prime}\right)$ should be provided.
2.1. Calculating the Sensitivity Coefficients. The two codes which perform the criticality calculations in this paper use different methods for calculating the sensitivity coefficients necessary to carry out the uncertainty quantification:

(i) SCALE-6.1 uses the Adjoint-Weighted Technique.

(ii) MCNPX-2.7e uses the Differential Operator Technique.

2.1.1. SCALE-6.1-The Adjoint-Weighted Technique. The Adjoint-Weighted Technique is used in SCALE-6.1 inside the TSUNAMI sequence, and the theory applied is stated in the SAMS module manual [2, Section F22]. Every sensitivity coefficient is calculated as a sum of two terms: explicit and implicit, as presented in

$$
\left(S_{k}\right)_{\text {complete }}=\left(S_{k}\right)_{\text {explicit }}+\left(S_{k}\right)_{\text {implicit }} .
$$

The explicit term, $\left(S_{k}\right)_{\text {explicit }}$, is calculated using the AdjointWeighted Technique (called adjoint-based perturbation in the SAMS manual), based on the perturbation $\delta k$ of the $k_{\text {eff }}$ given in (8). There, $\phi$ is the neutron flux of the problem defined by $[A-\lambda B] \phi=0$, where $A$ is the operator that represents all of the transport equations except for the fission term, $B$ is the operator that represents the fission term, and $\lambda$ represents the eigenvalues where the largest one is $1 / k_{\text {eff }} \cdot \phi^{\dagger}$ is the adjoint neutron flux of the adjoint problem defined as $\left[A^{\dagger}-\lambda B^{\dagger}\right] \phi^{\dagger}=0$ where $A^{\dagger}$ and $B^{\dagger}$ are the adjoint operators corresponding to $A$ and $B . \delta A$ and $\delta B$ are the perturbed operators due to a perturbation in the cross sections:

$$
\frac{\delta k_{\mathrm{eff}}}{k_{\mathrm{eff}}}=-\frac{\left\langle\phi^{\dagger}(\delta A-\lambda \delta B) \phi\right\rangle}{\left\langle\phi^{\dagger}(\lambda \delta B) \phi\right\rangle} .
$$

The implicit term, $\left(S_{k}\right)_{\text {implicit }}$, is calculated in order to take into account the effect on $k_{\text {eff }}$ of perturbing one cross section that affects the resonance-shielded values of other cross sections. This term appears because the transport calculations should be done using groupwise cross sections which require an initial self-shielding adjustment.

In any TSUNAMI sequence, the forward and adjoint transport problems are solved in order to calculate the neutron flux and its adjoint using XSDRNPM module for TSUNAMI-1D, NEWT module for TSUNAMI-2D, and KENO-V.a or KENO-VI for TSUNAMI-3D. Then, the SAMS module is applied for calculating the sensitivity coefficients for every energy-group reaction cross section. The sensitivity to the average number of neutrons per fission, $\bar{v}$, and to the fission spectrum, $\chi$, is estimated. Also, SAMS module performs the uncertainty quantification on the $k_{\text {eff }}$ due to the cross section uncertainties.

2.1.2. MCNPX-2.7e-The Differential Operator Technique. The Differential Operator Technique is applied in MCNPX2.7e to calculate the change $\Delta k_{\text {eff }}$ due to a perturbation in a cross section, $\Delta \sigma$, by means of the PERT card. It is based on the Taylor series expansion as presented in (9), where $\sigma_{x}$ 


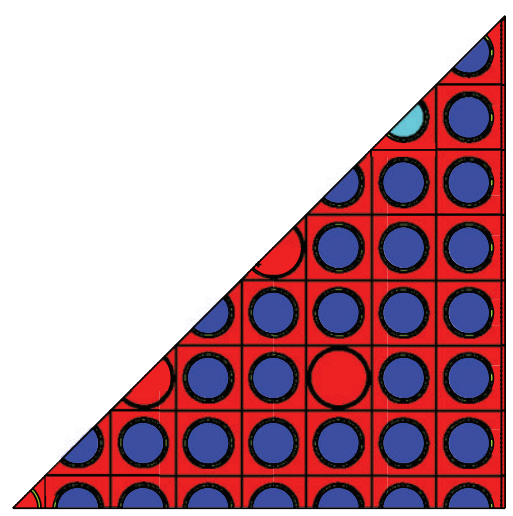

(a) Unrodded

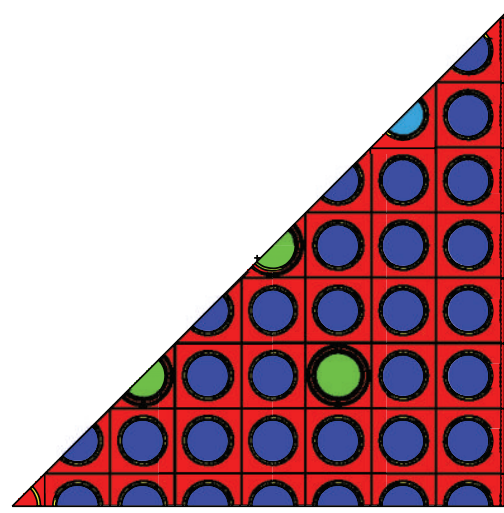

(b) Rodded

Figure 1: Geometrical model implemented in MCNPX-2.7e for 1/8 of the $15 \times 15$ TMI fuel assembly.

is the cross section perturbed, $\sigma_{x, 0}$ is its nominal value, and $\Delta \sigma=\sigma_{x}-\sigma_{x, 0}$, is the perturbation:

$$
\begin{aligned}
\Delta k_{\mathrm{eff}}= & k_{\mathrm{eff}}\left(\sigma_{x}\right)-k_{\mathrm{eff}}\left(\sigma_{x, 0}\right)=\left(\left.\frac{\partial k}{\partial \sigma}\right|_{\sigma_{x, 0}} \Delta \sigma\right)_{1 \mathrm{st}} \\
& +\left(\left.\frac{1}{2} \frac{\partial^{2} k}{\partial \sigma^{2}}\right|_{\sigma_{x, 0}}(\Delta \sigma)^{2}\right)_{2 \mathrm{nd}}+\cdots \\
= & {\left[\Delta k_{\mathrm{eff}}\right]_{\text {PERT }, 1 \mathrm{st}}+\left[\Delta k_{\mathrm{eff}}\right]_{\text {PERT }, 2 \mathrm{nd}}+\cdots }
\end{aligned}
$$

The first derivative means the derivative of the probability of the random walk occurring. The second one is the derivative of the tally response itself. There is an additional derivative for the changes in the fission source distribution that MCNPX2.7e does not take into account, but methodologies about how to calculate it have been developed in [4, 7-9] showing also its effect. In $[4,10]$, it was pointed out that because the scattering affects the fission source spatial distribution more than capture, the sensitivity to scattering cross sections could be wrong. Other limitations are stated in the MCNPX manual [3, Section 5.2.2].

For the sensitivity coefficient, only the first derivative is required. Using the first term provided by the PERT card (using the keyword METHOD=+2), the sensitivity coefficient is calculated with (10), just dividing by $k_{\mathrm{eff}, 0}$ and $p_{x}$, which is the variation of the cross section from $\sigma_{x}=\sigma_{x, 0}\left(1+p_{x}\right)$. Because $\left[\Delta k_{\mathrm{eff}}\right]_{\text {PERT,1st }}$ is lineal with respect to the size of the perturbation $p_{x}$, so choosing one value for $p_{x}$ is trivial:

$$
S_{i}=\frac{1}{k_{0} p_{x}}\left[\Delta k_{\mathrm{eff}}\right]_{\mathrm{PERT}, 1 \mathrm{st}}
$$

The statistical uncertainty is propagated to the sensitivity coefficient by (11), obtaining the relative standard deviation:

$$
\frac{\sqrt{\operatorname{var}\left(S_{i}\right)}}{S_{i}}=\sqrt{\frac{\operatorname{var}\left(\left[\Delta k_{\text {eff }}\right]_{\text {PERT,1st }}\right)}{\left[\Delta k_{\text {eff }}\right]_{\text {PERT,1st }}^{2}}+\frac{\operatorname{var}\left(k_{0}\right)}{k_{0}^{2}}} .
$$

TABLE 1: Reaction cross sections perturbed using PERT cards in the MCNPX-2.7e calculations.

\begin{tabular}{lc}
\hline Reaction & Isotopes \\
\hline$\sigma(n, n)$ & $152,154,155,156,157,158,160 \mathrm{Gd},{ }^{238} \mathrm{U},{ }^{107,109} \mathrm{Ag},{ }^{113} \mathrm{Cd},{ }^{115} \mathrm{In}$ \\
$\sigma\left(n, n^{\prime}\right)$ & ${ }^{238} \mathrm{U}$ \\
$\sigma(n, f)$ & ${ }^{235,238} \mathrm{U}$ \\
$\sigma(n, \gamma)$ & $152,154,155,156,157,158,160 \mathrm{Gd},{ }^{238} \mathrm{U},{ }^{107,109} \mathrm{Ag},{ }^{113} \mathrm{Cd},{ }^{115} \mathrm{In}$ \\
\hline
\end{tabular}

\section{PWR Calculations on Exercise I-2}

3.1. Specifications and Modeling Exercise I-2. Exercise I-2 [2, Chapter 3] proposes uncertainty propagation of the input parameters through the lattice physics to output variables for different Light Water Reactors (LWRs). Here, only the first one of three proposed test problems regarding PWR geometry is carried out. It consists in propagating the cross section uncertainties in a $2 \mathrm{D}$ fuel assembly model with reflective boundary conditions which will be used later as the standard model for fuel assembly cross-section generation in PWR core analysis.

The specifications of the fuel assembly are given in $[1$, Section 3.2]. It is a $15 \times 15 \mathrm{TMI}$ fuel assembly model which contains gadolinia pins as burnable poison. Also, the specifications of the control rods are found there. The definition of the pin cell is the same as used in Exercise I-1 [1, Section 2.4]. The fuel assembly configuration is presented in Figure 1(a) for the unrodded case and in Figure 1(b) for the rodded case. The fuel pins are in blue, the gadolinia pins in light blue, the control rods in green, cladding in yellow, and water in red.

The criticality calculations for the $15 \times 15$ TMI fuel assembly are carried out at Hot Zero Power (HZP) and at Hot Full Power (HFP) conditions for both cases, unrodded and rodded, together with uncertainty quantification on $k_{\text {eff }}$ due to the cross section uncertainties.

3.1.1. SCALE-6.1. For the criticality calculations with sensitivity analysis and uncertainty quantification of SCALE-6.1, the TSUNAMI-2D sequence is chosen. There, the NEWT 
TABLE 2: $k_{\text {eff }}$ values for the different states of the $15 \times 15$ TMI fuel assembly obtained by MCNPX-2.7e and SCALE-6.1, taking the SCALE results as the reference for the difference in $\mathrm{pcm}$.

\begin{tabular}{lcccc}
\hline & & & & Rodded \\
& HZP & Unrodded & HFP & HFP \\
\hline MCNPX-2.7.e & $1.41768 \pm 16 \mathrm{pcm}$ & $1.40441 \pm 16 \mathrm{pcm}$ & $1.07386 \pm 22 \mathrm{pcm}$ & $1.06146 \pm 25 \mathrm{pcm}$ \\
SCALE-6.1 & 1.41227 & 1.39802 & 1.07160 & 1.05834 \\
\hline Difference & $383 \mathrm{pcm}$ & $457 \mathrm{pcm}$ & $211 \mathrm{pcm}$ & $295 \mathrm{pcm}$ \\
\hline
\end{tabular}

TABLE 3: Comparison of the uncertainty contribution to $k_{\text {eff }}$ of each variance-covariance matrix of the reaction pair presented in the first two columns given in rel.std.dev.(\%) calculated by SCALE-6.1 and MCNPX-2.7e for unrodded case.

(a) HZP

\begin{tabular}{|c|c|c|c|c|c|c|}
\hline \multicolumn{2}{|c|}{ Reaction } & \multicolumn{2}{|c|}{ Reaction } & \multirow{2}{*}{$\frac{\text { MCNP-2.7e }}{0.23145}$} & \multirow{2}{*}{$\begin{array}{c}\text { SCALE-6.1 } \\
0.24582\end{array}$} & \multirow{2}{*}{$\begin{array}{l}\text { Ratio } \\
0.942\end{array}$} \\
\hline$\overline{{ }^{238} \mathrm{U}}$ & $(n, \gamma)$ & ${ }^{238} \mathrm{U}$ & $(n, \gamma)$ & & & \\
\hline${ }^{235} \mathrm{U}$ & $(n, \gamma)$ & ${ }^{235} \mathrm{U}$ & $(n, \gamma)$ & 0.19674 & 0.19970 & 0.985 \\
\hline${ }^{235} \mathrm{U}$ & $(n, f)$ & ${ }^{235} \mathrm{U}$ & $(n, \gamma)$ & 0.10733 & 0.10895 & 0.985 \\
\hline${ }^{238} \mathrm{U}$ & $\left(n, n^{\prime}\right)$ & ${ }^{238} \mathrm{U}$ & $\left(n, n^{\prime}\right)$ & 0.09078 & 0.09455 & 0.960 \\
\hline${ }^{235} \mathrm{U}$ & $(n, f)$ & ${ }^{235} \mathrm{U}$ & $(n, f)$ & 0.08368 & 0.08500 & 0.985 \\
\hline${ }^{238} \mathrm{U}$ & $(n, f)$ & ${ }^{238} \mathrm{U}$ & $(n, f)$ & 0.01363 & 0.01366 & 0.998 \\
\hline${ }^{238} \mathrm{U}$ & $(n, n)$ & ${ }^{238} \mathrm{U}$ & $(n, \gamma)$ & 0.01215 & -0.00474 & -2.566 \\
\hline${ }^{238} \mathrm{U}$ & $(n, n)$ & ${ }^{238} \mathrm{U}$ & $(n, n)$ & 0.01185 & 0.02265 & 0.523 \\
\hline${ }^{157} \mathrm{Gd}$ & $(n, \gamma)$ & ${ }^{157} \mathrm{Gd}$ & $(n, \gamma)$ & 0.00645 & 0.00683 & 0.945 \\
\hline${ }^{155} \mathrm{Gd}$ & $(n, \gamma)$ & ${ }^{155} \mathrm{Gd}$ & $(n, \gamma)$ & 0.00476 & 0.00488 & 0.977 \\
\hline${ }^{238} \mathrm{U}$ & $(n, f)$ & ${ }^{238} \mathrm{U}$ & $(n, \gamma)$ & 0.00246 & 0.00257 & 0.957 \\
\hline${ }^{156} \mathrm{Gd}$ & $(n, \gamma)$ & ${ }^{156} \mathrm{Gd}$ & $(n, \gamma)$ & 0.00129 & 0.00128 & 1.006 \\
\hline${ }^{238} \mathrm{U}$ & $(n, n)$ & ${ }^{238} \mathrm{U}$ & $(n, f)$ & -0.00116 & 0.00065 & -1.787 \\
\hline \multirow[t]{2}{*}{${ }^{238} \mathrm{U}$} & $(n, n)$ & ${ }^{238} \mathrm{U}$ & $\left(n, n^{\prime}\right)$ & -0.00476 & -0.00504 & 0.944 \\
\hline & & & Total & 0.34577 & 0.35940 & 0.962 \\
\hline${ }^{235} \mathrm{U}$ & $\bar{\nu}$ & ${ }^{235} \mathrm{U}$ & $\bar{v}$ & - & 0.26937 & \\
\hline \multirow[t]{2}{*}{${ }^{235} \mathrm{U}$} & $\chi$ & ${ }^{235} \mathrm{U}$ & $x$ & - & 0.08531 & \\
\hline & & & & ALE & 0.46441 & 0.774 \\
\hline
\end{tabular}

(b) HFP

\begin{tabular}{|c|c|c|c|c|c|c|}
\hline \multicolumn{2}{|c|}{ Reaction } & \multicolumn{2}{|c|}{ Reaction } & \multirow{2}{*}{$\begin{array}{c}\text { MCNP-2.7e } \\
0.23793\end{array}$} & \multirow{2}{*}{$\begin{array}{c}\text { SCALE-6.1 } \\
0.25293\end{array}$} & \multirow{2}{*}{$\begin{array}{l}\text { Ratio } \\
0.941\end{array}$} \\
\hline${ }^{238} \mathrm{U}$ & $(n, \gamma)$ & ${ }^{238} \mathrm{U}$ & $(n, \gamma)$ & & & \\
\hline${ }^{235} \mathrm{U}$ & $(n, \gamma)$ & ${ }^{235} \mathrm{U}$ & $(n, \gamma)$ & 0.19738 & 0.20025 & 0.986 \\
\hline${ }^{235} \mathrm{U}$ & $(n, f)$ & ${ }^{235} \mathrm{U}$ & $(n, \gamma)$ & 0.10697 & 0.10860 & 0.985 \\
\hline${ }^{238} \mathrm{U}$ & $\left(n, n^{\prime}\right)$ & ${ }^{238} \mathrm{U}$ & $\left(n, n^{\prime}\right)$ & 0.09808 & 0.09962 & 0.985 \\
\hline${ }^{235} \mathrm{U}$ & $(n, f)$ & ${ }^{235} \mathrm{U}$ & $(n, f)$ & 0.08333 & 0.08470 & 0.984 \\
\hline${ }^{238} \mathrm{U}$ & $(n, n)$ & ${ }^{238} \mathrm{U}$ & $\left(n, n^{\prime}\right)$ & 0.02302 & -0.00562 & -4.093 \\
\hline${ }^{238} \mathrm{U}$ & $(n, n)$ & ${ }^{238} \mathrm{U}$ & $(n, \gamma)$ & 0.01634 & 0.00943 & 1.732 \\
\hline${ }^{238} \mathrm{U}$ & $(n, f)$ & ${ }^{238} \mathrm{U}$ & $(n, f)$ & 0.01407 & 0.01412 & 0.997 \\
\hline${ }^{238} \mathrm{U}$ & $(n, n)$ & ${ }^{238} \mathrm{U}$ & $(n, n)$ & 0.01358 & 0.02520 & 0.539 \\
\hline${ }^{157} \mathrm{Gd}$ & $(n, \gamma)$ & ${ }^{157} \mathrm{Gd}$ & $(n, \gamma)$ & 0.00653 & 0.00693 & 0.943 \\
\hline${ }^{155} \mathrm{Gd}$ & $(n, \gamma)$ & ${ }^{155} \mathrm{Gd}$ & $(n, \gamma)$ & 0.00480 & 0.00496 & 0.967 \\
\hline${ }^{238} \mathrm{U}$ & $(n, f)$ & ${ }^{238} \mathrm{U}$ & $(n, \gamma)$ & 0.00259 & 0.00272 & 0.953 \\
\hline${ }^{156} \mathrm{Gd}$ & $(n, \gamma)$ & ${ }^{156} \mathrm{Gd}$ & $(n, \gamma)$ & 0.00135 & 0.00132 & 1.023 \\
\hline \multirow[t]{2}{*}{${ }^{238} \mathrm{U}$} & $(n, n)$ & ${ }^{238} \mathrm{U}$ & $(n, f)$ & -0.00137 & 0.00070 & -1.959 \\
\hline & & & Total & 0.35331 & 0.36607 & 0.965 \\
\hline${ }^{235} \mathrm{U}$ & $\bar{\nu}$ & ${ }^{235} \mathrm{U}$ & $\bar{v}$ & - & 0.26834 & \\
\hline \multirow[t]{2}{*}{${ }^{235} \mathrm{U}$} & $x$ & ${ }^{235} \mathrm{U}$ & $x$ & - & 0.08823 & \\
\hline & & & \multicolumn{2}{|c|}{ Total SCALE } & 0.46984 & 0.779 \\
\hline
\end{tabular}


TABLE 4: Comparison of the uncertainty contribution to $k_{\text {eff }}$ of each variance-covariance matrix of the reaction pair presented in the first two columns given in rel.std.dev.(\%) calculated by SCALE-6.1 and MCNPX-2.7e for rodded case.

(a) HZP

\begin{tabular}{|c|c|c|c|c|c|c|}
\hline \multicolumn{2}{|c|}{ Reaction } & \multicolumn{2}{|c|}{ Reaction } & \multirow{2}{*}{$\begin{array}{c}\text { MCNP-2.7e } \\
0.21670\end{array}$} & \multirow{2}{*}{$\begin{array}{c}\text { SCALE-6.1 } \\
0.23086\end{array}$} & \multirow{2}{*}{$\begin{array}{l}\text { Ratio } \\
0.939\end{array}$} \\
\hline${ }^{238} \mathrm{U}$ & $(n, \gamma)$ & ${ }^{238} \mathrm{U}$ & $(n, \gamma)$ & & & \\
\hline${ }^{235} \mathrm{U}$ & $(n, \gamma)$ & ${ }^{235} \mathrm{U}$ & $(n, \gamma)$ & 0.17403 & 0.17602 & 0.989 \\
\hline${ }^{238} \mathrm{U}$ & $\left(n, n^{\prime}\right)$ & ${ }^{238} \mathrm{U}$ & $\left(n, n^{\prime}\right)$ & 0.16286 & 0.16089 & 1.012 \\
\hline${ }^{235} \mathrm{U}$ & $(n, f)$ & ${ }^{235} \mathrm{U}$ & $(n, \gamma)$ & 0.11027 & 0.11189 & 0.985 \\
\hline${ }^{235} \mathrm{U}$ & $(n, f)$ & ${ }^{235} \mathrm{U}$ & $(n, f)$ & 0.10708 & 0.10936 & 0.979 \\
\hline${ }^{109} \mathrm{Ag}$ & $(n, \gamma)$ & ${ }^{109} \mathrm{Ag}$ & $(n, \gamma)$ & 0.07569 & 0.07831 & 0.967 \\
\hline${ }^{107} \mathrm{Ag}$ & $(n, \gamma)$ & ${ }^{107} \mathrm{Ag}$ & $(n, \gamma)$ & 0.06786 & 0.06965 & 0.974 \\
\hline${ }^{115} \mathrm{In}$ & $(n, \gamma)$ & ${ }^{115} \mathrm{In}$ & $(n, \gamma)$ & 0.03640 & 0.03627 & 1.003 \\
\hline${ }^{238} \mathrm{U}$ & $(n, f)$ & ${ }^{238} \mathrm{U}$ & $(n, f)$ & 0.02242 & 0.02237 & 1.002 \\
\hline${ }^{238} \mathrm{U}$ & $(n, n)$ & ${ }^{238} \mathrm{U}$ & $(n, \gamma)$ & 0.01812 & 0.01185 & 1.529 \\
\hline${ }^{238} \mathrm{U}$ & $(n, n)$ & ${ }^{238} \mathrm{U}$ & $(n, n)$ & 0.01357 & 0.02340 & 0.580 \\
\hline${ }^{157} \mathrm{Gd}$ & $(n, \gamma)$ & ${ }^{157} \mathrm{Gd}$ & $(n, \gamma)$ & 0.00747 & 0.00817 & 0.915 \\
\hline${ }^{113} \mathrm{Cd}$ & $(n, \gamma)$ & ${ }^{113} \mathrm{Cd}$ & $(n, \gamma)$ & 0.00744 & 0.00738 & 1.008 \\
\hline${ }^{155} \mathrm{Gd}$ & $(n, \gamma)$ & ${ }^{155} \mathrm{Gd}$ & $(n, \gamma)$ & 0.00556 & 0.00584 & 0.952 \\
\hline${ }^{107} \mathrm{Ag}$ & $(n, n)$ & ${ }^{107} \mathrm{Ag}$ & $(n, n)$ & 0.00347 & 0.00042 & 8.193 \\
\hline${ }^{238} \mathrm{U}$ & $(n, f)$ & ${ }^{238} \mathrm{U}$ & $(n, \gamma)$ & 0.00322 & 0.00337 & 0.955 \\
\hline${ }^{115} \mathrm{In}$ & $(n, n)$ & ${ }^{115} \mathrm{In}$ & $(n, n)$ & 0.00310 & 0.00164 & 1.888 \\
\hline${ }^{156} \mathrm{Gd}$ & $(n, \gamma)$ & ${ }^{156} \mathrm{Gd}$ & $(n, \gamma)$ & 0.00162 & 0.00154 & 1.050 \\
\hline${ }^{109} \mathrm{Ag}$ & $(n, n)$ & ${ }^{109} \mathrm{Ag}$ & $(n, n)$ & 0.00145 & 0.00031 & 4.695 \\
\hline${ }^{238} \mathrm{U}$ & $(n, n)$ & ${ }^{238} \mathrm{U}$ & $(n, f)$ & -0.00139 & 0.00088 & -1.579 \\
\hline \multirow[t]{2}{*}{${ }^{238} \mathrm{U}$} & $(n, n)$ & ${ }^{238} \mathrm{U}$ & $\left(n, n^{\prime}\right)$ & -0.03164 & $-\mathbf{0 . 0 0 7 5 7}$ & 4.178 \\
\hline & & & Total & 0.37315 & 0.38518 & 0.969 \\
\hline${ }^{235} \mathrm{U}$ & $\bar{v}$ & ${ }^{235} \mathrm{U}$ & $\bar{v}$ & - & 0.25594 & \\
\hline \multirow[t]{2}{*}{${ }^{235} \mathrm{U}$} & $\chi$ & ${ }^{235} \mathrm{U}$ & $\chi$ & - & 0.13360 & \\
\hline & & & & ALE & 0.49199 & 0.783 \\
\hline
\end{tabular}

(b) HFP

\begin{tabular}{|c|c|c|c|c|c|c|}
\hline \multicolumn{2}{|c|}{ Reaction } & \multicolumn{2}{|c|}{ Reaction } & \multirow{2}{*}{$\begin{array}{c}\text { MCNP-2.7e } \\
0.22281\end{array}$} & \multirow{2}{*}{$\begin{array}{c}\text { SCALE-6.1 } \\
0.23783\end{array}$} & \multirow{2}{*}{$\begin{array}{l}\text { Ratio } \\
0.937\end{array}$} \\
\hline${ }^{238} \mathrm{U}$ & $(n, \gamma)$ & ${ }^{238} \mathrm{U}$ & $(n, \gamma)$ & & & \\
\hline${ }^{235} \mathrm{U}$ & $(n, \gamma)$ & ${ }^{235} \mathrm{U}$ & $(n, \gamma)$ & 0.17426 & 0.17639 & 0.988 \\
\hline${ }^{238} \mathrm{U}$ & $\left(n, n^{\prime}\right)$ & ${ }^{238} \mathrm{U}$ & $\left(n, n^{\prime}\right)$ & 0.16419 & 0.16909 & 0.971 \\
\hline${ }^{235} \mathrm{U}$ & $(n, f)$ & ${ }^{235} \mathrm{U}$ & $(n, \gamma)$ & 0.10985 & 0.11143 & 0.986 \\
\hline${ }^{235} \mathrm{U}$ & $(n, f)$ & ${ }^{235} \mathrm{U}$ & $(n, f)$ & 0.10703 & 0.10925 & 0.980 \\
\hline${ }^{109} \mathrm{Ag}$ & $(n, \gamma)$ & ${ }^{109} \mathrm{Ag}$ & $(n, \gamma)$ & 0.07689 & 0.07969 & 0.965 \\
\hline${ }^{107} \mathrm{Ag}$ & $(n, \gamma)$ & ${ }^{107} \mathrm{Ag}$ & $(n, \gamma)$ & 0.06925 & 0.07100 & 0.975 \\
\hline${ }^{115} \mathrm{In}$ & $(n, \gamma)$ & ${ }^{115} \mathrm{In}$ & $(n, \gamma)$ & 0.03707 & 0.03690 & 1.005 \\
\hline${ }^{238} \mathrm{U}$ & $(n, f)$ & ${ }^{238} \mathrm{U}$ & $(n, f)$ & 0.02313 & 0.02310 & 1.001 \\
\hline${ }^{238} \mathrm{U}$ & $(n, n)$ & ${ }^{238} \mathrm{U}$ & $(n, \gamma)$ & 0.02141 & 0.01592 & 1.345 \\
\hline${ }^{238} \mathrm{U}$ & $(n, n)$ & ${ }^{238} \mathrm{U}$ & $\left(n, n^{\prime}\right)$ & 0.01567 & -0.00854 & -1.835 \\
\hline${ }^{238} \mathrm{U}$ & $(n, n)$ & ${ }^{238} \mathrm{U}$ & $(n, n)$ & 0.01276 & 0.02589 & 0.493 \\
\hline${ }^{157} \mathrm{Gd}$ & $(n, \gamma)$ & ${ }^{157} \mathrm{Gd}$ & $(n, \gamma)$ & 0.00770 & 0.00825 & 0.934 \\
\hline${ }^{113} \mathrm{Cd}$ & $(n, \gamma)$ & ${ }^{113} \mathrm{Cd}$ & $(n, \gamma)$ & 0.00762 & 0.00748 & 1.019 \\
\hline${ }^{107} \mathrm{Ag}$ & $(n, n)$ & ${ }^{107} \mathrm{Ag}$ & $(n, n)$ & 0.00587 & 0.00043 & 13.758 \\
\hline${ }^{155} \mathrm{Gd}$ & $(n, \gamma)$ & ${ }^{155} \mathrm{Gd}$ & $(n, \gamma)$ & 0.00569 & 0.00592 & 0.961 \\
\hline${ }^{109} \mathrm{Ag}$ & $(n, n)$ & ${ }^{109} \mathrm{Ag}$ & $(n, n)$ & 0.00450 & 0.00031 & 14.391 \\
\hline${ }^{238} \mathrm{U}$ & $(n, f)$ & ${ }^{238} \mathrm{U}$ & $(n, \gamma)$ & 0.00341 & 0.00356 & 0.958 \\
\hline
\end{tabular}


(b) Continued.

\begin{tabular}{|c|c|c|c|c|c|c|}
\hline \multicolumn{2}{|c|}{ Reaction } & \multicolumn{2}{|c|}{ Reaction } & \multirow{2}{*}{$\frac{\text { MCNP-2.7e }}{\mathbf{0 . 0 0 2 6 2}}$} & \multirow{2}{*}{$\begin{array}{c}\text { SCALE-6.1 } \\
\mathbf{0 . 0 0 1 6 7}\end{array}$} & \multirow{2}{*}{$\begin{array}{l}\text { Ratio } \\
1.569\end{array}$} \\
\hline${ }^{115} \mathrm{In}$ & $(n, n)$ & ${ }^{115} \mathrm{In}$ & $(n, n)$ & & & \\
\hline${ }^{156} \mathrm{Gd}$ & $(n, \gamma)$ & ${ }^{156} \mathrm{Gd}$ & $(n, \gamma)$ & 0.00166 & 0.00159 & 1.048 \\
\hline \multirow[t]{2}{*}{${ }^{238} \mathrm{U}$} & $(n, n)$ & ${ }^{238} \mathrm{U}$ & $(n, f)$ & -0.00151 & 0.00094 & -1.599 \\
\hline & & & Total & 0.37973 & 0.39380 & 0.964 \\
\hline${ }^{235} \mathrm{U}$ & $\bar{v}$ & ${ }^{235} \mathrm{U}$ & $\bar{v}$ & - & 0.25451 & \\
\hline \multirow[t]{2}{*}{${ }^{235} \mathrm{U}$} & $\chi$ & ${ }^{235} \mathrm{U}$ & $\chi$ & - & 0.13788 & \\
\hline & & & \multicolumn{2}{|c|}{ Total SCALE } & 0.49966 & 0.788 \\
\hline
\end{tabular}
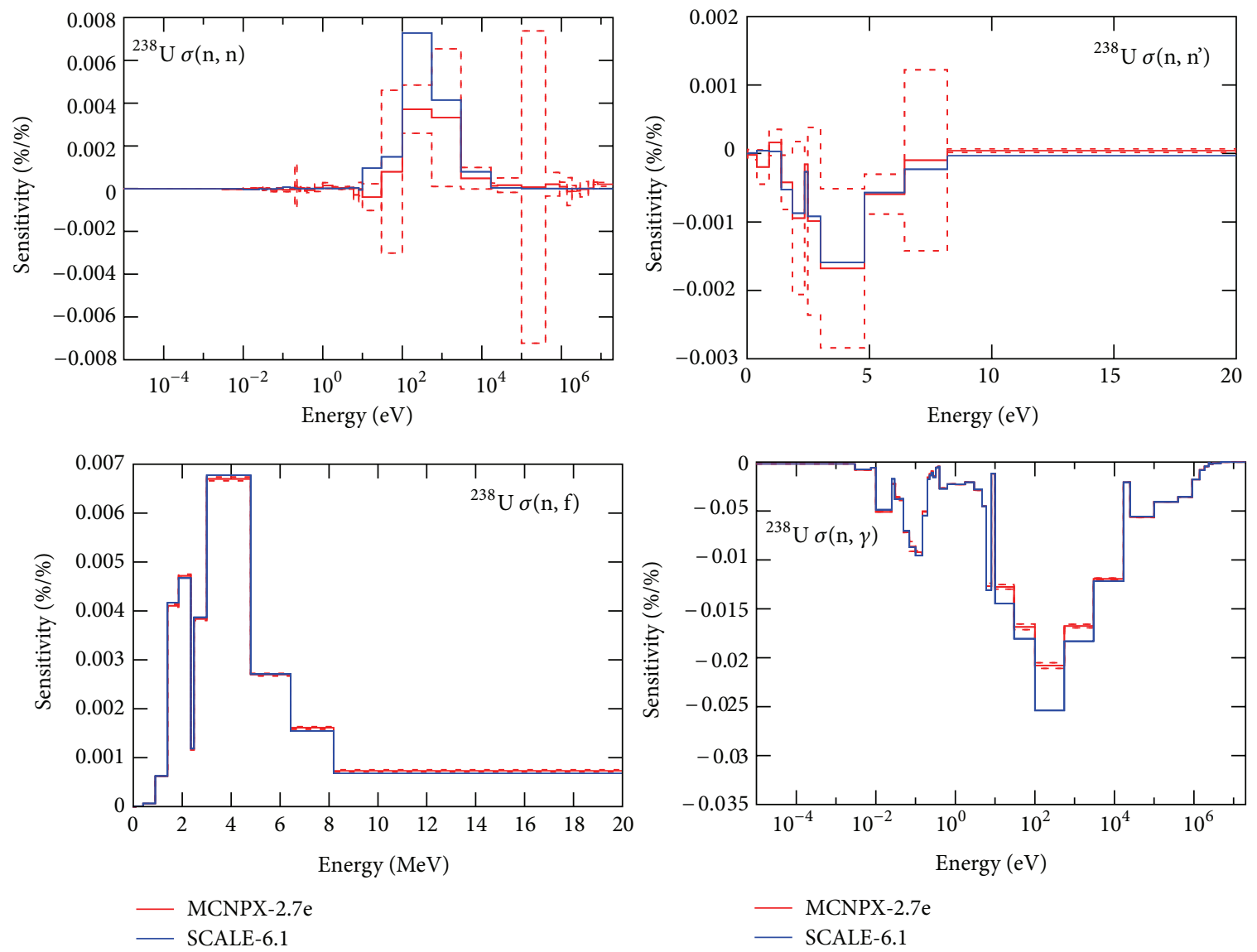

FIGURE 2: Sensitivity profiles of ${ }^{238} \mathrm{U}$ reaction cross sections calculated by MCNPX-2.7e and SCALE-6.1 for unrodded case at HZP.

module [2, Section F21] is used for solving the forward and adjoint transport problems. NEWT is an algorithm for twodimensional analysis in nonorthogonal geometries combining a step characteristics approach plus discrete ordinates. The 238 energy group cross section library of the ENDF/BVII.0 [2, Section M4] is used with NEWT (using the keyword v7-238). Then, the SAMS module [2, Section F22] is invoked to calculate the sensitivity coefficients in 238 energy groups. Because SCALE-6.1 provides a set of covariance matrices to perform the uncertainty quantification, "SCALE Nuclear Data Covariance Library" [2, Section M19] that is given in 44 energy group [2, Table M4.2.1], the sensitivity profiles are collapsed from 238 to 44 energy group structure inside SAMS, but the latter profile is not supplied to the user. But collapsing to 44 energy groups can be done with the VIBE tool [11].
3.1.2. MCNPX-2.7e. For the criticality calculations of MCNPX-2.7e, the KCODE card [3, Section 5.2.2] is applied and activated only for neutrons. The ENDF/B-VII.0 cross section library is used, taking the processed cross section files for MCNPX from [12]. Because the cross section library used for ${ }^{107} \mathrm{Ag}$ in [12] comes from JEFF-3.1.1, the ${ }^{107} \mathrm{Ag}$ ENDF/B-VII.0 cross section file is processed and included in the set of cross section libraries used by MCNPX-2.7e to substitute the previous one.

After preparing the inputs for the criticality calculations, PERT cards are added in order to calculate the sensitivity coefficients of $k_{\text {eff }}$ to the reaction cross sections given in Table 1.

To calculate the same sensitivity coefficients as SCALE6.1, the keyword that sets to which reaction cross section is 

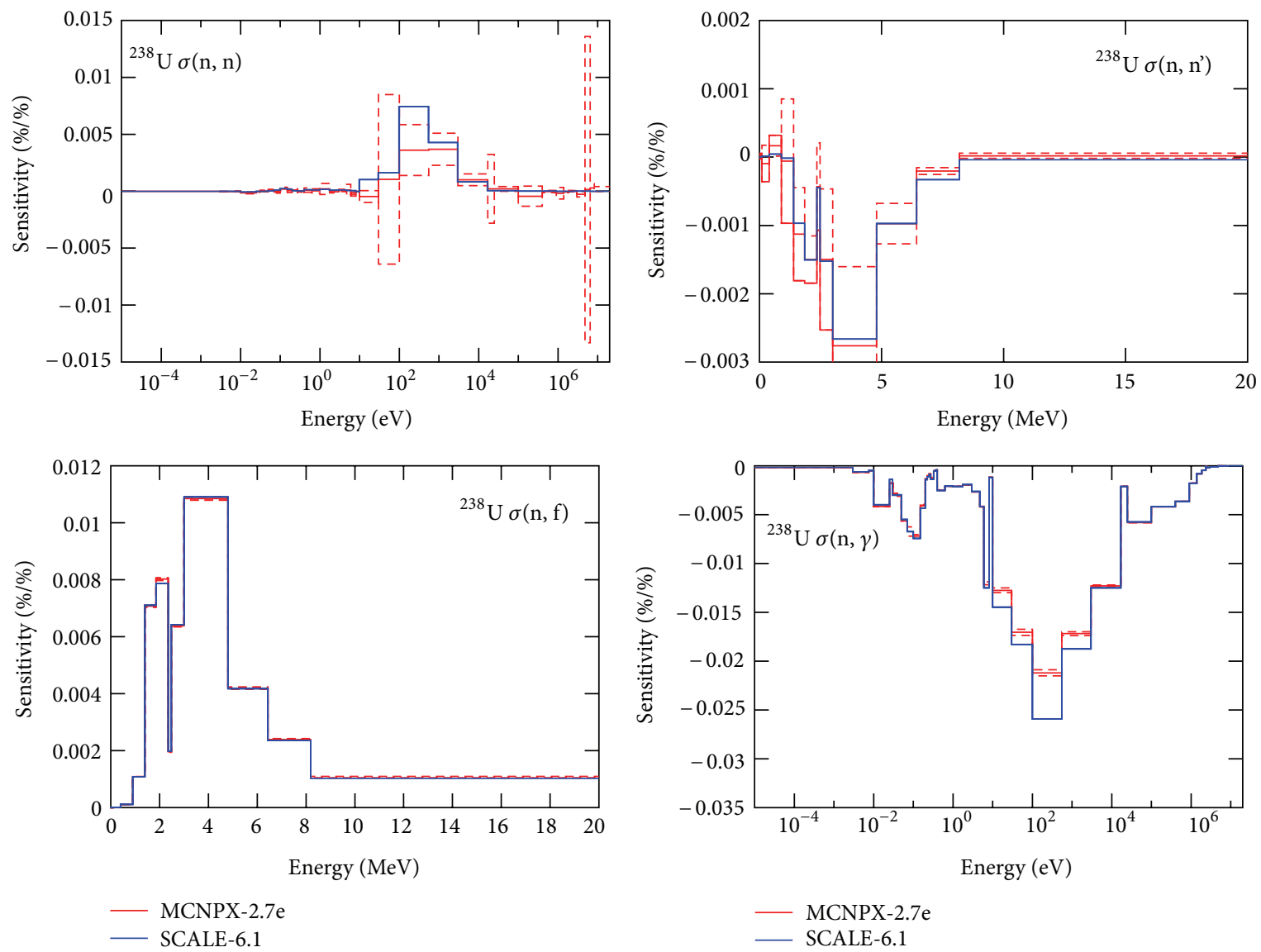

FIGURE 3: Sensitivity profiles of ${ }^{238} \mathrm{U}$ reaction cross sections calculated by MCNPX-2.7e and SCALE-6.1 for rodded case at HZP.

assigned the perturbation on the $k_{\text {eff }}$, RXN, is specified taking into account the equivalences between RXN and the identifiers of the SCALE reaction cross sections presented in [4, Table $\mathrm{V}]$. In order to compare the sensitivity profiles provided by SCALE-6.1 and MCNPX-2.7e, the PERT cards should use the same 44 energy group structure used by SCALE-6.1. $p_{x}$ is set to 100 to calculate the sensitivity coefficients by (10).

Once the sensitivity coefficients are calculated, the "SCALE Nuclear Data Covariance Library" is processed by the VIEWCVX code (provided as a module of ERRORJ code [13]) to obtain the relative covariance matrix of the reactions given in Table 1 . Then, the "sandwich formula" given in (6) is calculated, obtaining the uncertainty on $k_{\text {eff }}$.

3.2. Comparison of $k_{\text {eff }}$ Values. The $k_{\text {eff }}$ values are presented in Table 2. MCNPX-2.7e provides larger values than SCALE6.1 for all cases, but the difference between codes is reduced when going from unrodded to rodded case. These differences appear due to the use of multigroup cross-sections or continuous energy cross-sections, the different methodologies used in each code to solve the neutron transport equations, and the different processing codes used for preparing the cross section data files: NJOY v99.364 for MCNPX-2.7e and AMPX for SCALE-6.1.
3.3. Comparison of the $k_{\text {eff }}$ Uncertainty. The uncertainty results obtained by SCALE-6.1 and MCNPX-2.7e are presented in the tables: for the unrodded fuel assembly at HZP (Table 3(a)), and at HFP (Table 3(b)); for the rodded case at HZP (Table 4(a)), and at HFP (Table 4(b)). These tables present in the first two columns the reaction pair of the variance-covariance matrix that contributes to the $k_{\text {eff }}$ uncertainty. The contribution is given in rel.std.dev.(\%) in the third and fourth columns. Only the contributions with ||rel.std.dev.(\%)|| $>0.001$ are presented, sorted in descending order. The square root of the sum of the square rel.std.dev. provides the total uncertainty in $k_{\text {eff }}$ as rel.std.dev.(\%). The ratio between MCNPX-2.7e and SCALE-6.1 values is given for each contribution and for the total values. Two more rows are added to show the importance of the ${ }^{235} \mathrm{U} \bar{\nu}$ and ${ }^{235} \mathrm{U} \chi$ uncertainty contributions to $k_{\text {eff }}$, calculated by SCALE-6.1. The last row shows the total uncertainty given by SCALE6.1, taking into account all the possible reactions for which there is uncertainty information, and the ratio to the total uncertainty provided by MCNPX-2.7e.

There is a good agreement between MCNPX-2.7e and SCALE-6.1 results except when the $(n, n)$ reaction cross section is involved. There is a contribution in the HZP unrodded case in which the $(n, n)$ reaction is involved and both codes remain in good agreement: the ${ }^{238} \mathrm{U}(n, n)-{ }^{238} \mathrm{U}$ 

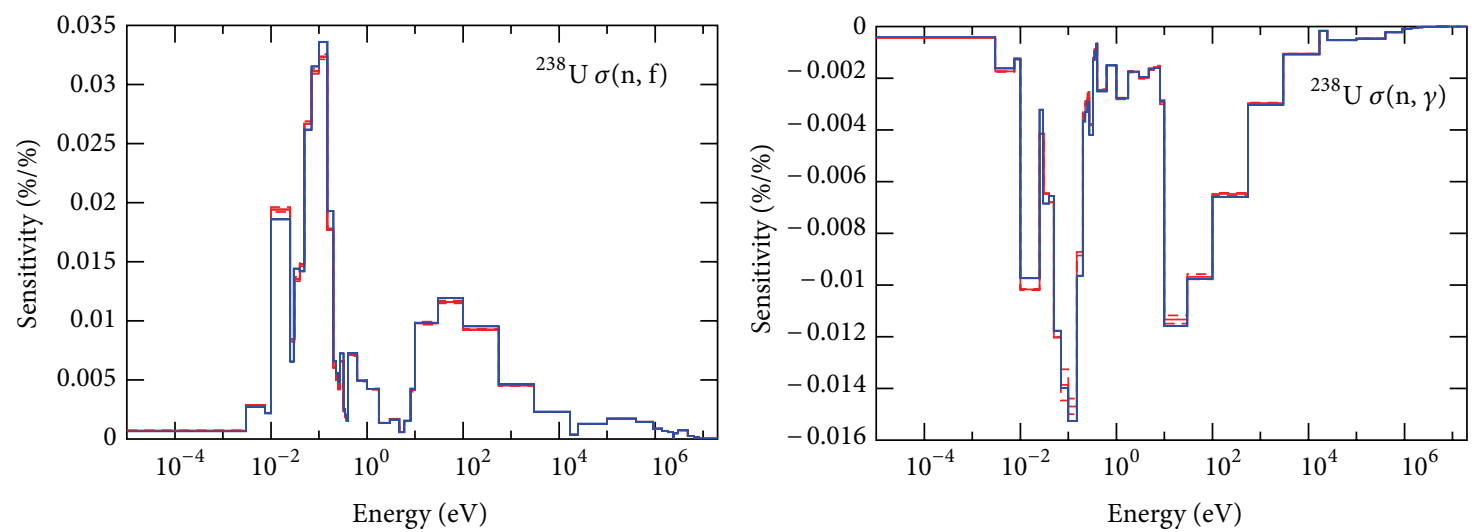

(a) Unrodded
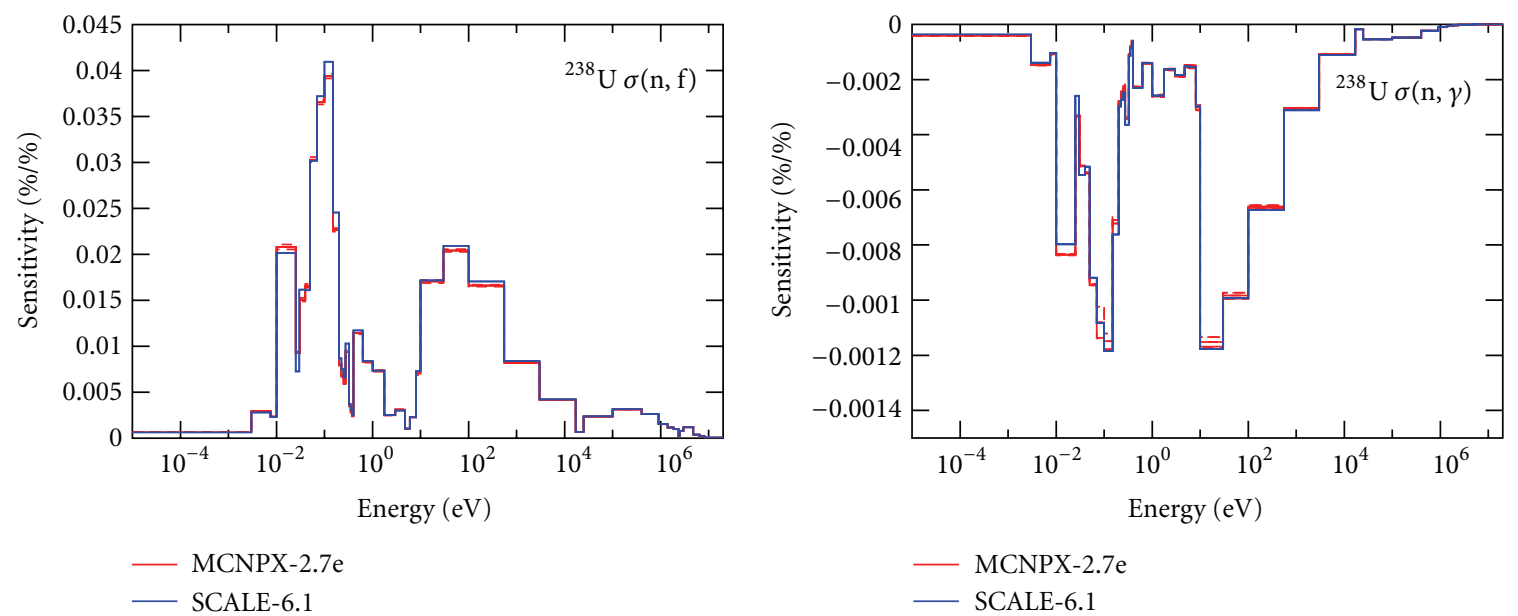

(b) Rodded

FIGURE 4: Sensitivity profiles of ${ }^{235} \mathrm{U}$ reaction cross sections calculated by MCNPX-2.7e and SCALE-6.1 for unrodded and rodded cases at HZP.

$\left(n, n^{\prime}\right)$ covariance matrix contribution. But this result is obtained by chance, because as showed later, there are large differences in the ${ }^{238} \mathrm{U}(n, n)$ sensitivity profile between both codes, while for the ${ }^{238} \mathrm{U}\left(n, n^{\prime}\right)$ sensitivity profile there is almost no difference. Therefore, after applying the "sandwich formula," the contribution given by each code is almost the same due to the combination of the differences in the ${ }^{238} \mathrm{U}$ $(n, n)$ sensitivity profile.

However, the $(n, n)$ reactions are included in the total values given by MCNPX; they have no impact because their contribution to the total is negligible.

It is necessary to implement more PERT cards in MCNPX-2.7e, because only with the ones calculated, the total uncertainty in the $k_{\text {eff }}$ is being underestimated around a $23 \%$. This $23 \%$ comes mainly because MCNPX-2.7e is not able to calculate the sensitivities to ${ }^{235} \mathrm{U} \bar{\nu}$ and ${ }^{235} \mathrm{U} \chi$ yet. But this capability could be implemented as presented in [14].

The differences between HZP and HFP are explained later through the comparison of the sensitivity profiles, because the variance-covariance matrices used in the "sandwich formula" do not change between cases.
3.4. Comparison of Sensitivity Profiles. The sensitivity profiles of ${ }^{238} \mathrm{U}$ reaction cross sections calculated by MCNPX-2.7e and SCALE-6.1 are presented in Figures 2 and 3, and for ${ }^{235} \mathrm{U}$ reaction cross sections in Figure 4, both for unrodded and rodded cases at HZP. MCNPX values are given in red, with dashed lines for their statistical uncertainty because of the Monte Carlo approach of solving the transport problem, and SCALE ones are in blue.

There is good agreement between all reaction cross sections except for $(n, n)$. Special remark should be done for the large statistical uncertainty on the $\left(n, n^{\prime}\right)$ reaction while its mean value remains in good agreement with the SCALE6.1 values. For the other isotope-reaction cross sections, the same trend is observed: good agreement for all reaction cross section except for the $(n, n)$ reaction. These kinds of differences in $(n, n)$ reaction were found before in [4], where the scattering reactions did not agree well with the SCALE results because of the deficiencies of the Differences Operator Technique used in the PERT card.

The temperature effect on the sensitivity profiles is analysed. Only for the unrodded case there is a noticeable change 
TABLE 5: Largest integrated sensitivity coefficients of the reaction cross sections for the unrodded case at HZP and HFP sorted in descending order provided by SCALE-6.1.

(a) HZP

\begin{tabular}{|c|c|c|}
\hline \multicolumn{2}{|c|}{ Reaction } & Int.sen.coef. \\
\hline${ }^{235} \mathrm{U}$ & $\bar{v}$ & $9.4533 E-01$ \\
\hline${ }^{235} \mathrm{U}$ & $(n, f)$ & $2.7342 E-01$ \\
\hline${ }^{238} \mathrm{U}$ & $(n, \gamma)$ & $-1.9175 E-01$ \\
\hline${ }^{1} \mathrm{H}$ & $(n, n)$ & $1.5701 E-01$ \\
\hline${ }^{235} \mathrm{U}$ & $(n, \gamma)$ & $-1.4756 E-01$ \\
\hline${ }^{238} \mathrm{U}$ & $\bar{v}$ & $5.4659 E-02$ \\
\hline${ }^{1} \mathrm{H}$ & $(n, \gamma)$ & $-4.5765 E-02$ \\
\hline${ }^{238} \mathrm{U}$ & $(n, f)$ & $2.6307 E-02$ \\
\hline${ }^{238} \mathrm{U}$ & $(n, n)$ & $1.4825 E-02$ \\
\hline
\end{tabular}

(b) HFP

\begin{tabular}{|c|c|c|}
\hline \multicolumn{2}{|c|}{ Reaction } & Int.sen.coef. \\
\hline${ }^{235} \mathrm{U}$ & $\bar{\nu}$ & $9.4404 E-01$ \\
\hline${ }^{235} \mathrm{U}$ & $(n, f)$ & $2.7389 E-01$ \\
\hline${ }^{238} \mathrm{U}$ & $(n, \gamma)$ & $-1.9659 E-01$ \\
\hline${ }^{1} \mathrm{H}$ & $(n, n)$ & $1.6365 E-01$ \\
\hline${ }^{235} \mathrm{U}$ & $(n, \gamma)$ & $-1.4806 E-01$ \\
\hline${ }^{238} \mathrm{U}$ & $\bar{v}$ & $5.5948 E-02$ \\
\hline${ }^{1} \mathrm{H}$ & $(n, \gamma)$ & $-4.4564 E-02$ \\
\hline${ }^{238} \mathrm{U}$ & $(n, f)$ & $2.7192 E-02$ \\
\hline${ }^{238} \mathrm{U}$ & $(n, n)$ & $1.5870 E-02$ \\
\hline
\end{tabular}

in the ${ }^{238} \mathrm{U}(n, \gamma)$ sensitivity profile; the sensitivity coefficients of the low energy groups are increased when going from HZP to HFP due to the Doppler broadening.

The effect of the control rods can be observed comparing Figures 2 (unrodded) and 3 (rodded). The ${ }^{238} \mathrm{U}(n, f)$ and $\left(n, n^{\prime}\right)$ reactions are affected: the sensitivity coefficients of the low energy groups are increased when going from unrodded to rodded case. The ${ }^{157} \mathrm{Gd}(n, \gamma)$ sensitivity coefficients follow the same trend: they are increased at low energies. The effect on ${ }^{235} \mathrm{U}$ reactions can be seen comparing Figures $4(\mathrm{a})$ and 4(b). The ${ }^{235} \mathrm{U}(n, f)$ sensitivity coefficients are increased in the whole range while the ${ }^{235} \mathrm{U}(n, \gamma)$ ones are decreased only at low energies, when changing from unrodded to rodded configuration. These changes provoke the variations observed in the contributions of these reactions to the $k_{\text {eff }}$ uncertainty.

Another source of difference, apart from the methodology used by each code to calculate the sensitivity coefficients, is that SCALE-6.1 provides the sensitivity profiles in 238 energy groups, while for MCNPX-2.7e the sensitivity profiles are calculated in 44 energy groups. Therefore, the energy group structure could lead to differences in between both codes.

3.5. Ranking the Importance of the Reactions. SCALE-6.1 provides a useful result: the integrated sensitivity coefficients of every reaction involved in the criticality calculations. It is the sum of the sensitivity coefficients over all energy groups
TABLE 6: Largest integrated sensitivity coefficients of the reaction cross sections for the rodded case at HZP and HFP sorted in descending order provided by SCALE-6.1.

(a) Rodded at HZP

\begin{tabular}{|c|c|c|}
\hline \multicolumn{2}{|c|}{ Reaction } & Int.sen.coef. \\
\hline${ }^{235} \mathrm{U}$ & $\bar{\nu}$ & $9.2720 E-01$ \\
\hline${ }^{235} \mathrm{U}$ & $(n, f)$ & $3.6753 E-01$ \\
\hline${ }^{1} \mathrm{H}$ & $(n, n)$ & $2.3743 E-01$ \\
\hline${ }^{238} \mathrm{U}$ & $(n, \gamma)$ & $-1.8097 E-01$ \\
\hline${ }^{235} \mathrm{U}$ & $(n, \gamma)$ & $-1.2798 E-01$ \\
\hline${ }^{238} \mathrm{U}$ & $\bar{v}$ & $7.1728 E-02$ \\
\hline${ }^{238} \mathrm{U}$ & $(n, f)$ & $4.3054 E-02$ \\
\hline${ }^{1} \mathrm{H}$ & $(n, \gamma)$ & $-3.3060 E-02$ \\
\hline${ }^{109} \mathrm{Ag}$ & $(n, \gamma)$ & $-2.1940 E-02$ \\
\hline${ }^{238} \mathrm{U}$ & $(n, n)$ & $1.6606 E-02$ \\
\hline${ }^{107} \mathrm{Ag}$ & $(n, \gamma)$ & $-1.3914 E-02$ \\
\hline${ }^{115} \mathrm{In}$ & $(n, \gamma)$ & $-1.2778 E-02$ \\
\hline
\end{tabular}

(b) Rodded at HFP

\begin{tabular}{|c|c|c|}
\hline \multicolumn{2}{|c|}{ Reaction } & Int.sen.coef. \\
\hline${ }^{235} \mathrm{U}$ & $\bar{v}$ & $9.2540 E-01$ \\
\hline${ }^{235} \mathrm{U}$ & $(n, f)$ & $3.6931 E-01$ \\
\hline${ }^{1} \mathrm{H}$ & $(n, n)$ & $2.4283 E-01$ \\
\hline${ }^{238} \mathrm{U}$ & $(n, \gamma)$ & $-1.8549 E-01$ \\
\hline${ }^{235} \mathrm{U}$ & $(n, \gamma)$ & $-1.2804 E-01$ \\
\hline${ }^{238} \mathrm{U}$ & $\bar{v}$ & $7.3537 E-02$ \\
\hline${ }^{238} \mathrm{U}$ & $(n, f)$ & $4.4462 E-02$ \\
\hline${ }^{1} \mathrm{H}$ & $(n, \gamma)$ & $-3.2066 E-02$ \\
\hline${ }^{109} \mathrm{Ag}$ & $(n, \gamma)$ & $-2.2270 E-02$ \\
\hline${ }^{238} \mathrm{U}$ & $(n, n)$ & $1.7615 E-02$ \\
\hline${ }^{107} \mathrm{Ag}$ & $(n, \gamma)$ & $-1.4158 E-02$ \\
\hline${ }^{115} \mathrm{In}$ & $(n, \gamma)$ & $-1.2976 E-02$ \\
\hline
\end{tabular}

of the same reaction. This value reflects how sensitive is the $k_{\text {eff }}$ to this reaction. Then, the reactions can be ranked as a function of their relevance to $k_{\text {eff }}$ with their integrated sensitivity coefficient.

With the sensitivity profiles provided by MCNPX-2.7e, the integrated sensitivity values can be calculated. Because comparing the integrated sensitivity coefficients is equivalent to compare sensitivity profiles, only the SCALE-6.1 results are shown in this section.

Table 5 presents the integrated sensitivity values for the unrodded case at HZP and HFP, while Table 6 shows the results for the rodded case at HZP and HFP. The most relevant reactions are the ones for ${ }^{235} \mathrm{U},{ }^{238} \mathrm{U}$, and ${ }^{1} \mathrm{H}$. In the rodded case, the $(n, \gamma)$ reactions of ${ }^{107} \mathrm{Ag},{ }^{109} \mathrm{Ag}$, and ${ }^{115} \mathrm{In}$ arise as important.

\section{Conclusions}

The propagation of cross section uncertainties in criticality calculations for a $15 \times 15$ TMI fuel assembly in two different configurations, unrodded and rodded, at two different states, HZP and HFP, has been performed using two 
different approaches carried out by each of the codes used: (i) MCNPX-2.7e-Differential Operator Technique and (ii) SCALE-6.1-Adjoint-Weighted Technique.

The two approaches have been presented and compared through this exercise. The $k_{\text {eff }}$ and its uncertainty due to cross-section uncertainties have been calculated, distinguishing between its different contributors. The most important ones for this exercise are ${ }^{235} \mathrm{U}-\bar{\nu}, \chi,(n, f),(n, \gamma),{ }^{238} \mathrm{U}-$ $\left(n, n^{\prime}\right),(n, f),(n, \gamma)$, and the covariance ${ }^{235} \mathrm{U}(n, f)-{ }^{235} \mathrm{U}$ $(n, \gamma)$. Additionally, the sensitivity coefficients and the integrated sensitivity coefficients have been calculated and compared.

In general, both approaches/codes are in good agreement, in spite of the differences in the $k_{\text {eff }}$ values. There are only two exceptions observed: one is for the contribution of the $(n, n)$ reaction cross sections which are not properly calculated by MCNPX-2.7e because the deficiencies of the PERT card to calculate the $(n, n)$ sensitivity coefficients, and the another is for the contribution of ${ }^{238} \mathrm{U}(n, n)-\left(n, n^{\prime}\right)$ covariance whose agreement between codes comes by chance because of the combination of the differences in the $(n, n)$ sensitivity profile. The good agreements between codes are corroborated by the comparison of the sensitivity profiles given by each code.

The inability of calculating the contribution due to $\bar{v}$ and $\chi$ values makes MCNPX-2.7e to underestimate the uncertainty in $k_{\text {eff }}$ at least in a $23 \%$ compared with SCALE-6.1. It is important to remark that the most relevant reactions are not always the main source of the uncertainty, such as the case of ${ }^{1} \mathrm{H}$ which is in the top list of reactions for which $k_{\text {eff }}$ is sensitive to, but it is not in the list of the most important contributions to the $k_{\text {eff }}$ uncertainty because of the low uncertainty of its reactions.

Thus, MCNPX-2.7e can deal with the uncertainty quantification problem as SCALE-6.1 does, but improvements should be done in the PERT card capabilities such as the proper calculation of the sensitivity coefficients of $(n, n)$ reaction cross sections, the inclusion of the perturbation due to the change in the fission source distribution, and the implementation of the perturbation of the $\bar{v}$ and $\chi$ values.

\section{Acknowledgments}

The research leading to these results has received funding from specific collaborative agreement P110530207 between CSN and UPM in the area of "uncertainty propagation in nuclear criticality safety." Also, this work is also partially supported by the Spanish Ministry of Education of Spain through the FPU Program for teaching and researching formation under Grant AP2009-1801 for the first author.

\section{References}

[1] K. Ivanova, M. Avramova, S. Kamerow et al., Benchmark For Uncertainty Analysis in Modeling (UAM) For Design, Operation and Safety Analysis of LWRs, NEA/NSC/DOC, 2012.

[2] Oak Ridge National Laboratory, "SCALE: a comprehensive modeling and simulation suite for nuclear safety analysis and design," ORNL/TM-2005/39, version 6.1, Radiation Safety
Information Computational Center, Oak Ridge National Laboratory as CCC-785, 2011.

[3] D. B. Pelowitz, "MCNPX user's manual," Tech. Rep. LA-CP-071473, Los Alamos National Laboratory, 2008.

[4] J. Favorite, "Eigenvalue sensitivity analysis using the MCNP5 perturbation capability," in Proceedings of the Nuclear Criticality Safety Division Topical Meeting on Realism, Robustness and the Nuclear Renaissance, pp. 245-255, American Nuclear Society, 2009.

[5] B. C. Kiedrowski and F. B. Brown, "Comparison of the Monte Carlo adjoint-weighted and di perturbation methods," Progress in Nuclear Science and Technology, vol. 2, pp. 836-841, 2011.

[6] D. G. Cacuci, Sensitivity and Uncertainty Analysis, Chapman Hall/CRC, London, UK, 2003.

[7] Y. Nagaya and F. B. Brown, "Estimation of the change in keffective due to perturbed fission source distribution in MCNP," in Proceedings of the ANS Mathematics \& Computation Topical Meeting (M\&C '03), Gatlinburg, Tenn, USA, 2003.

[8] Y. Nagaya and F. B. Brown, "Implementation of a method to estimate change in eigenvalue due to perturbed fission source distribution into MCNP," Tech. Rep. LA-UR-03-1387, Los Alamos National Laboratory, 2003.

[9] K. Raskach, "An improvement of the monte carlo generalized differential first- and second-order perturbations of fission source," Nuclear Science and Engineering, vol. 162, no. 2, pp. 158166, 2009.

[10] J. A. Favorite, "On the accuracy of the differential operator Monte Carlo perturbation method for eigenvalue problems," Technical Report LA-UR-09-4207, Los Alamos National Laboratory, 2009.

[11] B. Rearden and R. Lefebvre, "Getting Started with VIBE as a DICE Plug-in Module," Tech. Rep. ORNL/TM-2010/60, Oak Ridge National Laboratory, 2010.

[12] T. Viitanen and J. Leppanen, "ZZ SERPENT117-ACELIB, Continuous-energy X-sec lib., radioactive decay, fission yield data for SERPENT in ACE," Tech. Rep. NEA-1854, 2010.

[13] G. Chiba, "ERRORJ-a code to process neutron-nuclide reaction cross section covariance, version 2.3," JAEA-Data/Code 2007-007, Japan Atomic Energy Agency, 2007.

[14] R. L. Perel, "Sensitivities of $k_{\text {eff }}$ calculated with Monte-Carlo methods: theory and first results," Tech. Rep. JEF-DOC-1123, Nuclear Energy Agency, 2005. 


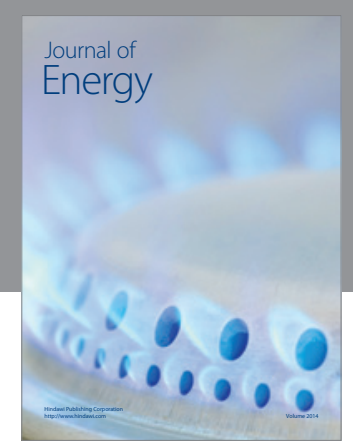

Journal of

Industrial Engineering
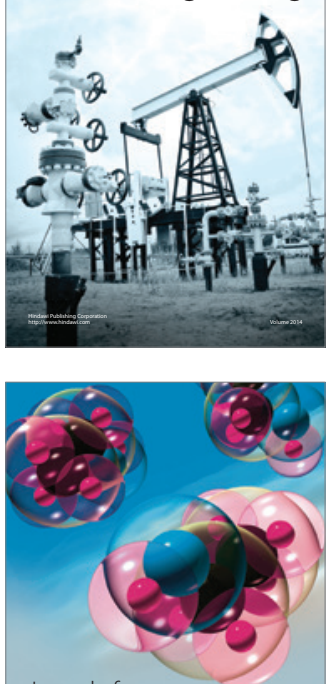

Fuels
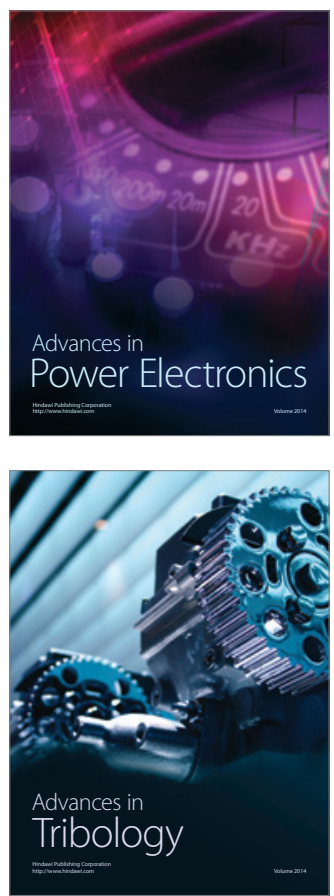

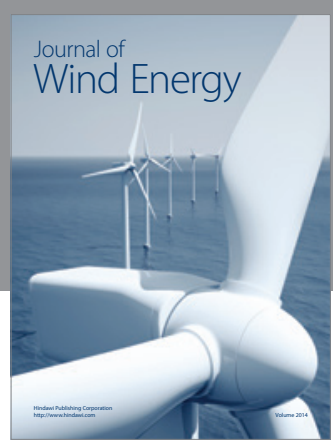

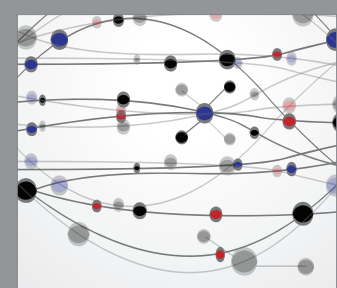

The Scientific World Journal

Submit your manuscripts at http://www.hindawi.com

Journal of

Structures
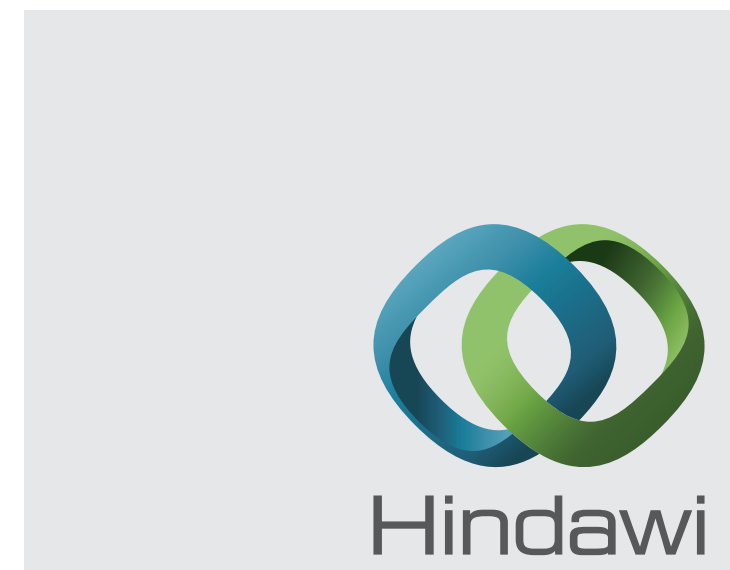

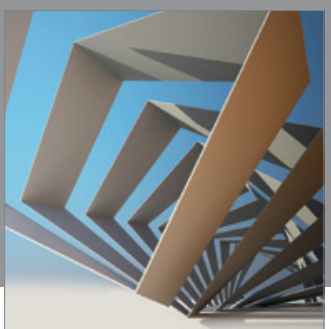

Rotating

Machinery
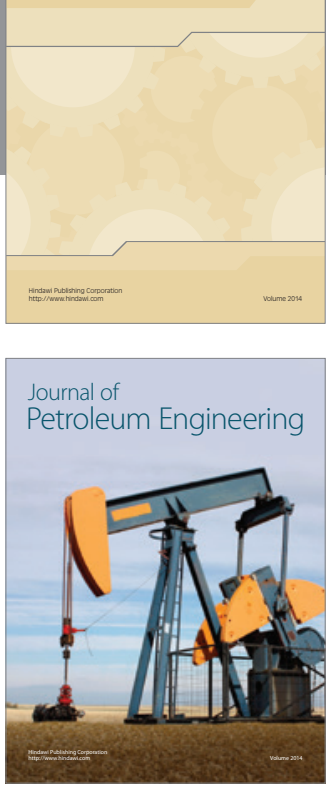

Journal of

Solar Energy
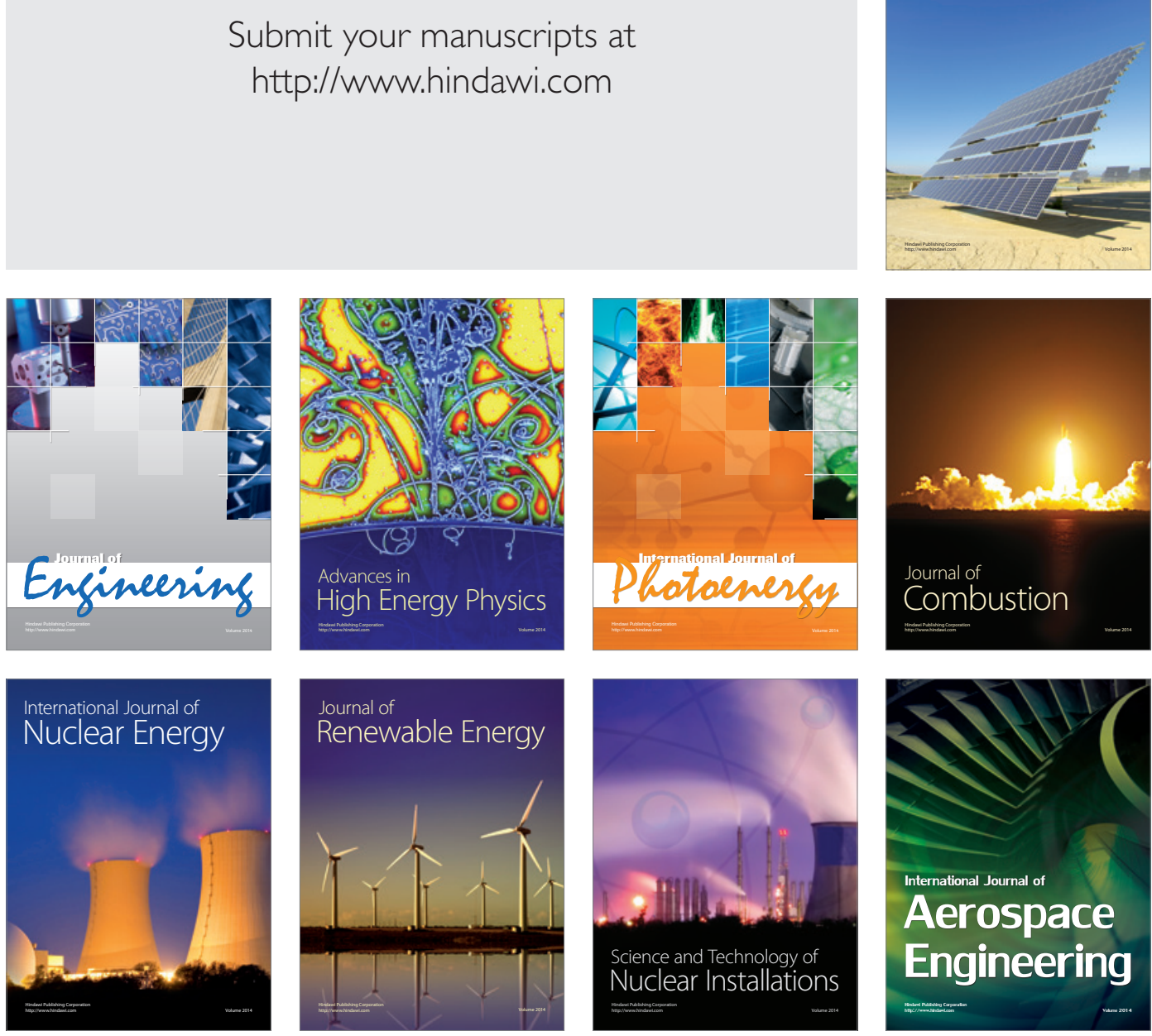